\title{
Would Changes to the 4 Hour Target Benefit Paediatric Procedural Sedation? A Service Review
} Charles G Stewart*, Poonam Patel, Nikita Mediratta

Paediatric Emergency Department, Chelsea and Westminster Healthcare NHS Fdn Trust, London, UK. *charles.stewart@chelwest.nhs.uk

*Corresponding Author: Charles G Stewart, Paediatric Emergency Department, Chelsea and Westminster Healthcare NHS Fdn Trust, London, UK.

Abstract

Aims: As Paediatric Emergency Departments (PED) become busier the number of children requiring painful procedures continues to increase. We aimed to evaluate a locally developed paediatric procedural sedations (PPS) service using ketamine for safety, efficiency and efficacy in the context of the national 4 hour operational target which is due to be revised this year in the UK

Methods: We enrolled consecutive patients over 12 months suitable for PPS ketamine and collected data for demographics, time to patient identification, time to ketamine administration, procedural duration and time to recovery. We also documented procedure outcome, adverse events, consent, ketamine dosage and baseline physiological observations

Results: From May 2017 to May 2018 ketamine PPS was performed on 36 patients with a mean age of 7 years (range 1.8 to 14.6 years). The most common procedure performed was manipulation of forearm fractures $(n=21,58 \%)$, followed by facial laceration repair $(n=10,28 \%)$. Total intravenous Ketamine dosages were; $1 \mathrm{mg} / \mathrm{kg}(\mathrm{n}=23,64 \%) 1.5 \mathrm{mg} / \mathrm{kg}(\mathrm{n}=10,28 \%)$ and $2 \mathrm{mg} / \mathrm{kg}(\mathrm{n}=3,8 \%)$. Average time to referral to surgical speciality was 33 minutes which improved from 40 minutes (pre Sept) to 27 minutes (post Sept). Average time to ketamine administration was 168 minutes, improved from 185 minutes (pre Sept) to 155 minutes (post Sept). The average time taken to complete procedures, all under 20 minutes, also decreased from 19 minutes (pre Sept) to 10 minutes (post Sept) averaging 15 minutes overall. The recovery time was similar throughout the study period. The overall average length of stay (LOS) was 284 minutes, improved from 297 minutes (pre Sept) to 274 minutes (post Sept) figure 3. 20 (55\%) of the 36 patients breached the 4 hour target. 10 (28\%) patients were admitted, 9 for further neurovascular observations and only 1 where the outcome of a procedure was unsatisfactory. There were no seminal untoward incidents in our study. Vomiting occurred in 4, there was 1 drug error and one patient required brief airway manoeuvres for decreased oxygen saturations

Conclusion: We have confirmed PPS ketamine service to be safe and further demonstrated good outcomes in procedures carried out. Despite improved efficiency in the study period the average LOS still falls outside the 4 hour target and a revision would be welcomed to reduce anxiety related to breaches in this group and encourage more uptake of PPS ketamine nationally

Keywords: paediatric emergency med, quality improvement, emergency care systems, efficiency.

\section{INTRODUCTION}

As paediatric emergency departments (PED) become busier the number of children requiring painful procedures will continue to increase ${ }^{1,2}$. PED staff aim to provide care that minimises pain and distress with analgesia and distraction, however, for more painful procedures sedation may be required as children are recognised to have a different response to pain and foreign environments than adults ${ }^{3,4}$. Paediatric Procedural Sedation (PPS) with ketamine has become the standard in many PED settings avoiding the need 
for general anaesthesia and unnecessary admission with delays in treatment ${ }^{5}$.

Ketamine has a favourable side effect profile with an excellent safety record since its first use for PPS in 1990. It has a quick onset of action, producing a dissociative state via blockade of $\mathrm{N}$-methyl D-aspartate (NMDA) receptors, peripheral $\mathrm{Na}+$ channels and $\mu$ opioid receptors providing sedation, amnesia, and analgesia while its adrenergic actions provide it with a safer cardiovascular profile compared to other anaesthetic agents. Ketamine has been described as the ideal agent for PPS in EDs4, 5.

Uptake of ketamine PPS in PEDs has been slower in the UK than in other healthcare zones, such as North America, and in some instances is slowing down ${ }^{6,9}$. With a $7.6 \%$ increase in PED attendances from 2015-2016 alone, conditions are sometimes too stretched to allocate resources for ketamine PPS with the possibility of 'breaching' the 4 hour target an added pressure ${ }^{7}$. The 4 hour target is a UK national operational standard whereby at least 95\% of patients attending ED should be admitted, transferred or discharged within four hours. With no such constraints in North America PED practitioners may be more inclined to perform ketamine PPS. The 4 hour target is under review and may be revised with a permissive increased length of stay (LOS) in selected low risk patient groups proposed ${ }^{8}$.Our department has over 40,000 paediatric attendances per year and is dedicated to providing ketamine PPS in an acceptable timeframe to support our existing successful 4 hour target performance.

We conducted a prospective audit of our ketamine PPS service to assess safety, quality and efficiency in the context of proposed changes to the 4 hour target.

\section{METHOD}

We evaluated our Ketamine PPS service in a busy London teaching hospital over 12 months from May 2017 to May 2018 enrolling consecutive patients over this time.

The child's injury was identified at triage as requiring a procedure which was predicted to take 20 minutes or less to complete. This was discussed with the senior medical staff member who, upon confirming injury suitability, made a joint decision with the senior nurse to initiate the ketamine PPS pathway based on staffing levels and patient volume.

The patient and their carer(s) were then approached by a PED doctor for risk evaluation and in the absence of any unstable disease (ASA $\leq 2$ ) or other contraindications (see figure 1) ketamine PPS was offered for injury management. They were given a patient information leaflet (PIL) and after 15 minutes were approached for their decision and to field any further questions. If consent was given, the relevant surgical specialty doctor was contacted, a peripheral intravenous (IV) cannula was inserted and the anaesthetic team notified of the intended procedure. The anaesthetic team was not required to be present for ketamine PPS but was made aware in case of significant airway complications. A minimum of 3 personnel were required for Ketamine PPS; the proceduralist, the sedationist and a staff nurse. Once ketamine was drawn up, a safety check list was carried out which included details of the procedure, attendees, availability of emergency equipment and any anticipated complications.

Prior to commencement of the procedure baseline measurements were taken for blood pressure, pulse, respiratory rate and oxygen saturations. These were repeated every 5 minutes throughout the procedure until full recovery. Ketamine $1 \mathrm{mg} / \mathrm{kg}$ was administered intravenously over 2 minutes and once an adequate dissociative state was achieved the procedure began. If required, more IV ketamine was administered in $0.5 \mathrm{mg} / \mathrm{kg}$ aliquots of up to a maximum of $1 \mathrm{mg} /$ $\mathrm{kg}$ extra. The patient was woken in a quiet nonthreatening environment and discharged with contact numbers and information on possible side effects once pre-procedure baseline levels of activity were regained

Retrospective data were collected for time of patient identification, time to ketamine administration, total time for procedure, time to recovery and LOS. Data were also collected for the procedure performed, demographics adverse events and outcome. We also audited the rate of informed, written consent and the performance of routine observations. 


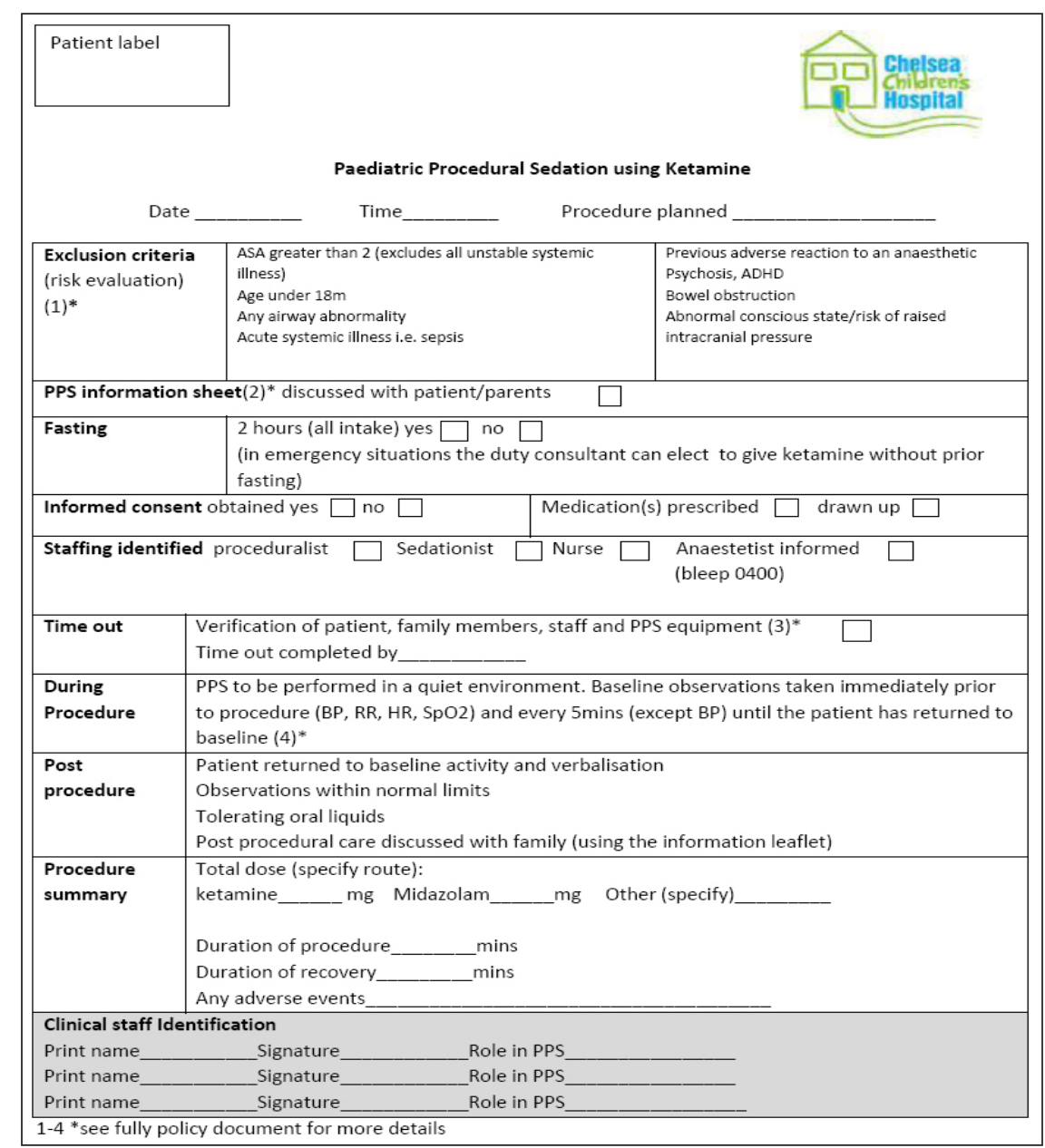

Fig1. Ketamine PPS proforma.

\section{RESULTS}

From May 2017 to May 2018 ketamine PPS was performed on 36 patients with a mean age of 7 years (range 1.8 to 14.6 years). The most common procedure performed was manipulation of forearm fractures $(n=21,58 \%)$, followed by facial laceration repair $(n=10$,
$28 \%$ ), figure 2 . Written consent was obtained for all patients with a Patient Information Leaflet provided on every occasion. Routine physiological observations were carried out prior to ketamine administration and every 5 minutes until full recovery in all patients. Total intravenous Ketamine dosages were; $1 \mathrm{mg} / \mathrm{kg}(\mathrm{n}=23$, $64 \%) 1.5 \mathrm{mg} / \mathrm{kg}(\mathrm{n}=10,28 \%)$ and $2 \mathrm{mg} / \mathrm{kg}(\mathrm{n}=3,8 \%)$.

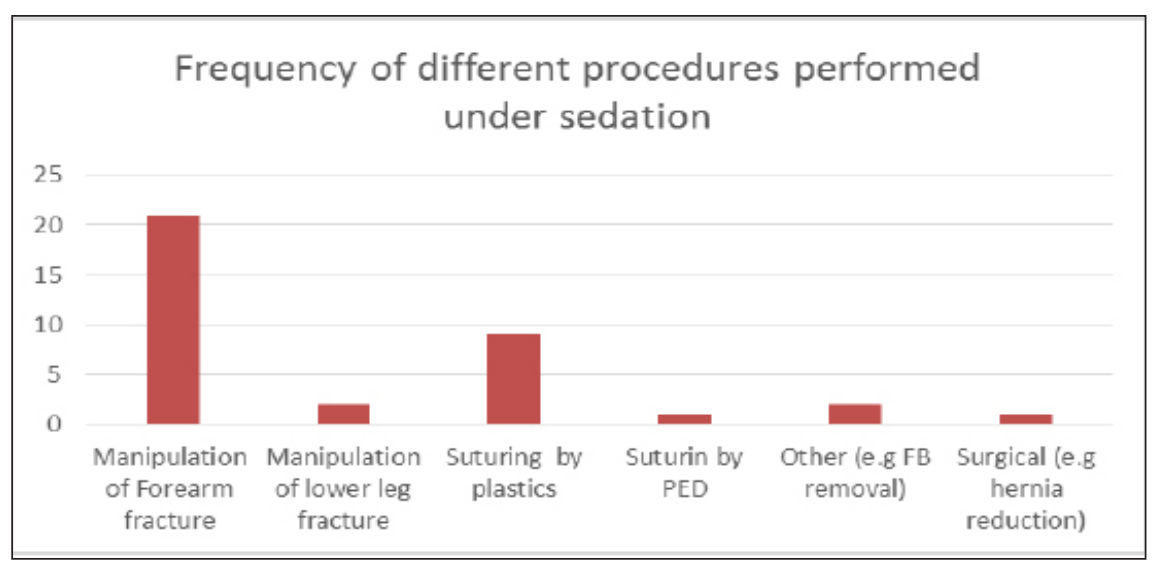

Fig 2. Procedures performed under ketamine PPS. 


\section{Pathway Performance}

We compared efficiency before and after the half-way point of our study (occurring September, 2017). The overall average time to referral to surgical speciality was 33 minutes which improved from 40 minutes (pre-September) to 27 minutes (post-September). Overall average time to ketamine administration was 168 minutes, improved from 185 minutes (pre-
September) to 155 minutes (post-September). The average time to complete procedures also decreased from 19 minutes (pre-September) to 10 minutes (post-September) averaging 15 minutes overall. The recovery time, 72 minutes, was similar throughout the study period. The overall average length of stay (LOS) was 284 minutes, improved from 297 minutes (preSeptember) to 274 minutes (post-September) figure 3.

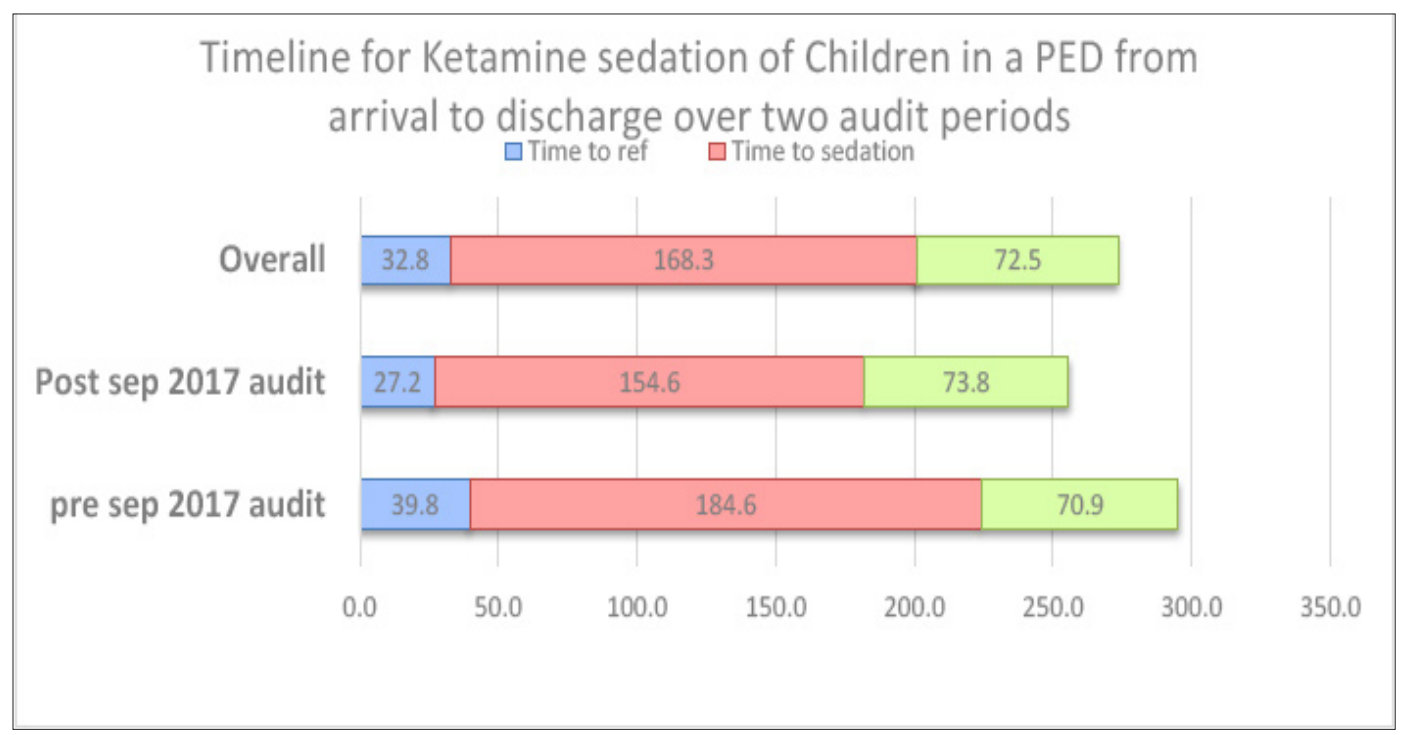

Fig 3. Patient pathway for ketamine PPS.

In $20(55 \%)$ patients the 4 hour target was breached, 9 (56\%) occurring post September. 10 (28\%) patients were admitted, 9 for further neurovascular observations and only 1 where the outcome of a procedure (manipulation of a tibia/fibula fracture) required further attention in theatre the next day. This fracture was predicted to require further surgery with PPS ketamine being used to address neurovascular concerns and thus avoid emergency late night surgery. This was achieved without any further complications.

There were no serious untoward incidents in this study. Adverse events did occur in $6(17 \%)$ procedures with vomiting $(n=4)$ the most common. There was 1 drug error in midazolam prescription (Midazolam has subsequently been removed from the protocol) and one patient required airway manoeuvres for decreased oxygen saturations. This patient quickly improved and the procedure was completed with a good outcome.

\section{DiscusSION}

Emergency departments (ED) worldwide are experiencing increasing workloads and paediatric emergency departments (PED) follow this trend. National ED waiting time averages in the UK have consistently breached the 4 hour target every year since 2013 with prolonged waiting times giving an indication of overcrowding and inefficiency ${ }^{10,11 .}$ This standard is applied equally to all patients no matter their acuity and if targets are not met financial penalties apply. This is not the only ED performance target but has the highest profile and introduces a time pressure to clinical practice. Ketamine PPS by definition involves well children undergoing noncomplex procedures and may be considered to be stifled by this unrealistic target. Others may advocate continuing within the current system with the focuses on time responsibility a positive.

Over the 12 month study period we demonstrated improvements in the entire patient pathway up to recovery time. We identified patients earlier, administered ketamine earlier and took less time in performing procedures. Results compare well with published data 12, 13,14 and demonstrate efficient team working not only within the PED but also from surgical department staff who committed to working 
towards an improvement in patient experience. With familiarity amongst nursing staff ketamine preparation time improved as did paperwork on risk identification, patient information, consent, time out and drug prescription. This was achieved without any extra resource allocation whereby all staff were asked to expand their clinical roles, supported by in-house education and training.

This study confirms ketamine's excellent safety profile with no serious incidents occurring in 36 patients. This mandates its consideration for use in paediatric procedures as children's response to pain is more intense than adults and is further intensified by a threatening alien environment ${ }^{12}$. Forced restraint is not an acceptable strategy and, with first line analgesics often inadequate for the planned procedures, an alternative must be sought ${ }^{6}$.

Ketamine has been described as the ideal agent for paediatric sedation in EDs as, in addition to being safe, it reduces pain, provides amnesia, avoids prolonged preoperative fasting and reduces time spent in hospital. It also has protective maintenance of both airway reflexes and sympathetic nervous system tone further adding to its appeal for use by non-anaesthetists. This, coupled with highly successful procedural outcomes explains why published patient feedback is overwhelmingly positive ${ }^{15,16}$. In our study 10 (28\%) patients were admitted primarily for observation with no 'failed' procedures, a similar rate to other studies 9. Whether admitted or not, Mitchell et al comment that ketamine PPS allows more timely definitive treatment which, assuming children and their carers want to spend as little time as possible in hospital, can be considered a positive ${ }^{18}$. The resources spared in avoiding theatre and admissions will produce cost savings to hospitals and also benefit patients and their families on travel costs, time off work and general inconvenience ${ }^{16}$.

Given the above validation, it is disappointing that ketamine PPS uptake in the UK is slow and in, some cases, reducing ${ }^{6,9}$. This is in light of increasing patient volume with up to $63 \%$ of PED presentations due to injuries ${ }^{17}$.

Despite becoming more efficient over the course of the study, reducing the average length of stay (LOS) by 23 minutes, the average LOS remained greater than 4 hours (274 minutes) and so breaches the national target. Our department is one of the few nationally to achieve the 4 hour target across the board and can absorb subsequent breaches, however, emergency clinicians under pressure from increased workload, staffing difficulties and overcrowding may be reluctant to adopt ketamine PPS with its predictably prolonged LOS.

The 4 hour target needs to be addressed as it oversimplifies the way in which performance in emergency departments is judged. A permissive increase in the LOS for ketamine PPS patients, who are well by definition, may encourage more PED units to adopting this service to the ultimate benefit of children who present with injuries

\section{REFERENCES}

[1] Adrian O'Dowd. Surge in child emergency admissions prompts new care standards BMJ 2017;356:j1480

[2] Under pressure. Safely managing increased demand in emergency departments. Care Quality Commission 2018

[3] Kennedy RM. The "ouchless emergency department" getting closer: advances in decreasing distress during painful procedures in the emergency department. Pediatr Clin North Am1999;46:1215-47

[4] Green SM et al. Ketamine sedation for pediatric procedures: Part 1, A prospective series. Ann Emerg Med. 1990 Sep;19(9):1024-32.

[5] Green SM et al. Ketamine is a safe, effective, and appropriate technique for emergency department paediatric procedural sedation Emergency Medicine Journal 2004;21:271-272.

[6] Neil S. Morton et al. Ketamine for procedural sedation and analgesia in pediatric emergency medicine: a UK perspective. Pediatric Anesthesia 2008 18: 25-29

[7] Adrian O'Dowd. Surge in child emergency admissions prompts new care standards BMJ 2017;356:j1480

[8] Gareth Iacobucci. NHS is to test scrapping the four hour A\&E target. BMJ 2019;364:11148

[9] Kidd, L.R e al. Paediatric procedural sedation using ketamine in a UK emergency department: a 7 year review of practice. British Journal of Anaesthesia , Volume 116, Issue 4, 518 - 523

[10] Under pressure. Safely managing increased demand in emergency departments. Care Quality Commission 2018 
Would Changes to the 4 Hour Target Benefit Paediatric Procedural Sedation? A Service Review

[11] What's going on with A\&E waiting times? | The King's Fund. 2019. https://www.kingsfund.org. uk/projects/urgent-emergency-care/urgentand-emergency-care-mythbusters

[12] Priestley SJ. Emerg Med (Fremantle). Ketamine sedation for children in the emergency department. 2001 Mar;13(1):82-90.

[13] Green SM et al. Ketamine sedation for pediatric procedures: Part 1, A prospective series. , Nakamura R, Johnson NE. Ann Emerg Med. 1990 Sep;19(9):1024-32.

[14] Jordan R.A comparison of ketamine sedation and general anaesthesia for manipulation of paediatric forearm fractures. Acta Orthop Belg. 2016 Dec;82(4):836-842
[15] Holloway VJ et al. Accident and emergency department led implementation of ketamine sedation in paediatric practice and parental response. Emergency Medicine Journal 2000; $17: 25-28$

[16] Anatole Vilhelm Wiik et al. Use of ketamine sedation for the management of displaced paediatric forearm fractures. World J Orthop. 2018 Mar 18; 9(3): 50-57

[17] A Downing et al. A study of childhood attendance at emergency departments in the West Midlands region. Emerg Med J. 2006 May; 23(5): 391-393

[18] Mitchell et all. Paediatric distal radial fracture manipulation: multicentre analysis of process times. Emerg Med J 26(1):41-2 January 2009.

Citation: Charles G Stewart, Poonam Patel, Nikita Mediratta. Would Changes to the 4 Hour Target Benefit Paediatric Procedural Sedation? A Service Review. Archives of Emergency Medicine and Intensive Care. 2019; 2(2): 22-27.

Copyright: (c) 2019 Charles G Stewart, Poonam Patel, Nikita Mediratta. This is an open access article distributed under the Creative Commons Attribution License, which permits unrestricted use, distribution, and reproduction in any medium, provided the original work is properly cited. 